\section{Reseña \\ Un pueblo unido por la fe} Espacios de devoción

DOI: https://doi.org/10.18861/ania.2021.11.2.3200

Arq. Carla Nóbile

nobile.carla@gmail.com

ORCID: https://orcid.org/0000-0002-1309-2034

Cómo citar:

Nóbile, C. (2021). Un pueblo unido por la fe. Anales de Investigacion en Arquitectura, 11(2). Recuperado a partir de https://revistas.ort.edu.uy/anales-de-investigacion-en-
El libro de la Dra. Virginia Flores Sasso y el Dr. Esteban Prieto Vicioso (2020) propone posicionar los espacios de com investigación la crisis sanitaria internacional actual lo autores revisitan la arquitectura religiosa, la historia de sus edificios y sus congregaciones en la actual Republica Dominicana con varias finalidades. Difundir el rico acervo de arquitectura religiosa presente en la isla, como patrimonio culturat y arquitectónico de la nación, promocionar dichos espacios de devoción dentro del circuito turístico a partir de una publicación clara en su relato y de gran calidad gráfica, y por último, cepensar el rol de la religión como refugio de sociedad ante situaciones de crisis como la que atraviesa el mundo en la actualidad.

La Dra. Flores Sasso y el Dr. Prieto Vicioso cuentan ambos con una amplia trayectoria y vinculación con los estudios referidos a la historia colonial en la zona del Caribe. Sus trabajos de investigación sobre la arquitectura colonial, las técnicas, los materiales, así como también las implicancias de orden político, social y cultural se extienden por más de una década y brindan un repertorio por demás interesante en cuanto a la presencia de estas estructuras en diversas regiones de los territorios del llamado Nuevo Mundo, en especial en la isla Española. Asimismo, la vinculación de ambos con la práctica arquitectónica y en especial con trabajos vinculados a la conservación, restauración y rehabilitación de edificios es de suma importancia para la investigación y análisis de las obras arquitectónicas del presente libro.

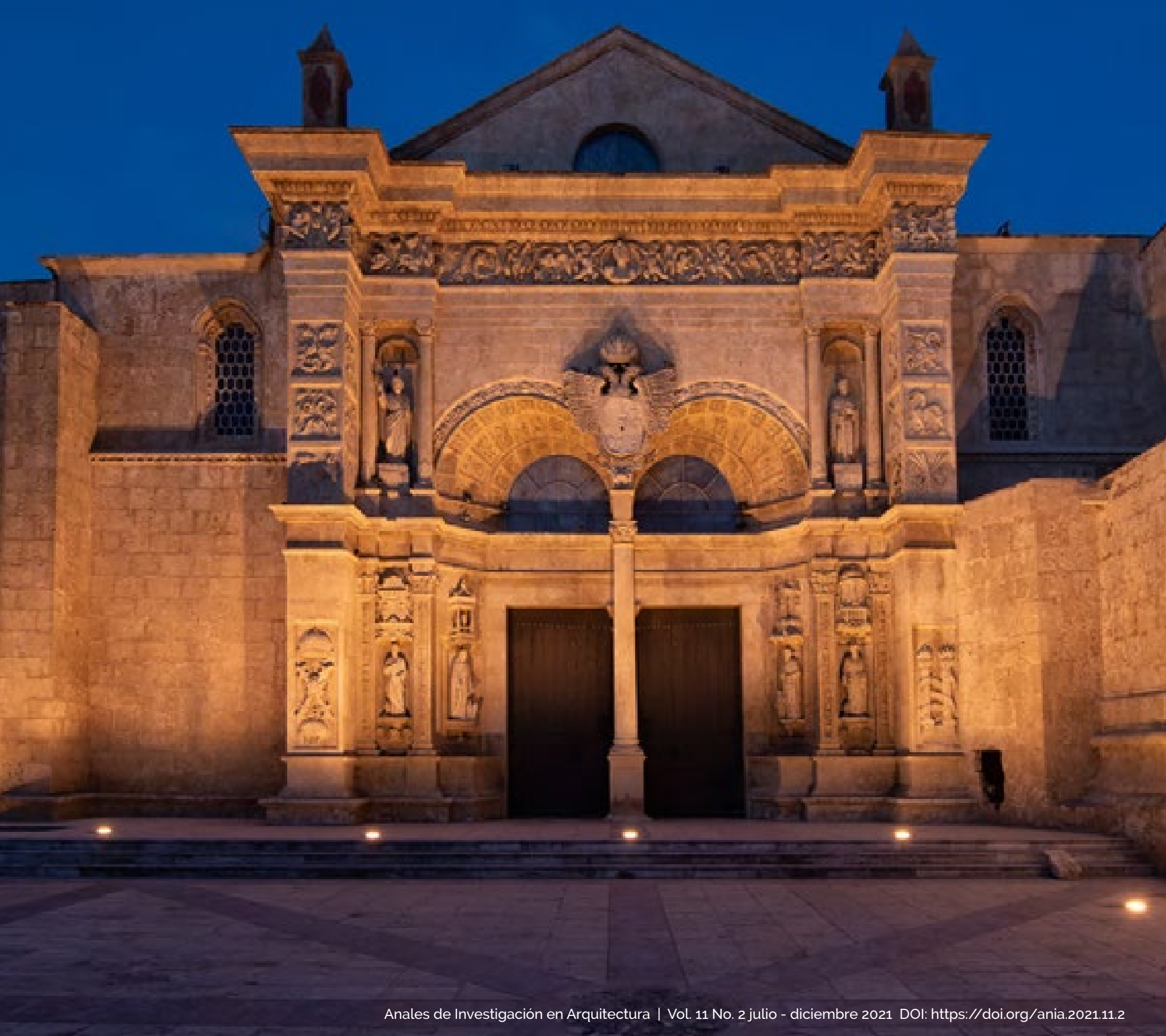




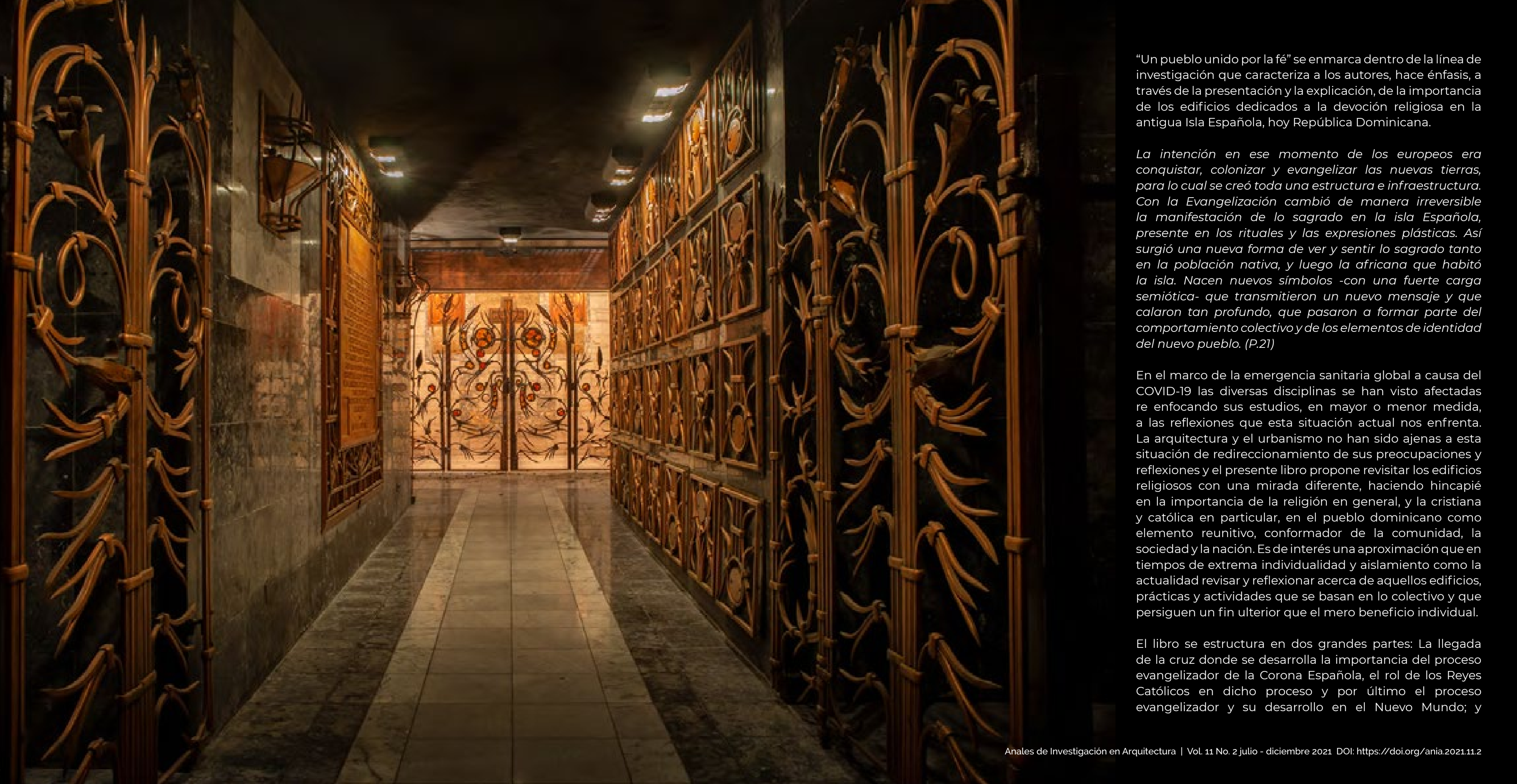


Espacios de devoción que a su vez se subdivide en catedrales, iglesias, conventos y monasterios católicos y otros espacios de devoción de la religión cristiana en República Dominicana.

Flores Sasso y Prieto Vicioso centran la primera parte de su narración en la importancia, desde los tiempos de la su narrion en la importancia, desde los tiempos de la conquista, coloni
de la religión.

En este contexto de profunda fe y fuerte convicción religiosa se inició la evangelización del Nuevo Mundo una tarea conjunta entre la Iglesia, la Corona y el pueblo, para lo que se creó toda una estructura: por una parte, la maquinaria política -Consejo de Indias, una parte, la maquinaria politica -Consejo de Indias y Patronato Real, entre otros-, el aparato fiscal -oficiales reales-, gobiernos locales y cabildos; y, por otra parte la eclesiástica con sus cabildos, arquidiócesis, diócesis, parroquias, conventos, monasterios y ermitas, entre otras, permitiendo esa inmensa labor misionera. (P.26)
El primer capítulo titulado "La llegada de la cruz" presenta con claridad el rol evangelizador del período colonia iniciado por los posteriormente denominados Reyes Católicos Isabel de Castillay Fernando de Aragón a patir de 1492. Continúa con los procesos que se llevaron a cabo en el Nuevo Mundo, poniendo el foco en la Isla Española una vez asentados los primeros colonos e iniciado el proceso de colonización, dominio y explotación de los nuevos territorios del imperio La presencia de la iglesia católica central a lo largo de todo el texto, pero no se deja de lado la importancia de otras prácticas religiosas cristianas que también aparecen tempranamente con la llegada de los protestantes a otras islas del Caribe y por tanto también a la Española. Esto se ve reflejado en la incorporación de obras de las distintas vertientes de la religión cristiana.

Sin embargo, desde finales del siglo XVI, comenzaron a llegar las ideas cristianas no católicas de la reforma protestante a la isla y a todo el Caribe. La Reforma entró y se propagó por las costas caribeñas a través de hombres de mar, contrabandistas, piratas, corsarios, filibusteros y bucaneros, quienes procedían de reinos e imperios europeos donde predominaba el protestantismo. (P.37)

En el segundo capítulo denominado "Espacios de devoción" los autores profundizan en el arte y la arquitectura de los edificios de devoción en el territorio dominicano El extenso catálogo de obras citadas da cuenta de la importancia de los edificios de carácter religioso en la isla desde comienzos del siglo XVI hasta ra actualidad La subidivisión en categorías de edificios según su programa permite al lector conocer e identificar la amplia variedad de edificios religiosos presentes en la isla y, por tanto, el peso real de la religión en pueblos, ciudades y la nación toda. Asimismo el análisis que los autores realizan de cada una de las obras permite, en la mayoría de ellas, conocer la historia desde la creación hasta hoy en día del edificio, su arquitectura, el arte que contiene y, por sobretodo la relación que ha guardado con su comunidad.

Los Reyes Católicos necesitaban fortalecer su imagen y presencia en los territorios que conformaban sus reinos, sobre todo en aquellos que se encontraban diseminados y distantes. Para estos fines, utilizaron la arquitectura y el arte no solo como sello distintivo de su reinado, sino como elemento unificador del territorio dentro de la diversidad geográfica y étnica, que representaba tanto la península ibérica como el Nuevo Mundo. (P.29) 

Señora de la Encarnación, Primada de América donde el conocimiento y participación de la Dra. Viricina Flores Saasso en el equipo de conservación y restauración de la misma nos permite un acercamiento y conocimiento de la obra no solamente desde si historia sino también desde sus elementos estructurales, decorativos y los procesos que se llevan a cabo en la contemporaneidad.

Las fuentes utilizadas a lo largo del trabajo dan cuenta de la seriedad, el conocimiento y buen manejo por parte de Flores Sasso y Prieto Vicioso del material existente, bien el material gráfico que se maneja para el análisis de las obras no incluye, casi en ningún caso, planos y se centra en alguno antiguos grabados pero mayormente en fotografías actuales, la que por su gran calidad permiten apreciar tanto los aspectos volumétricos y formales exteriores como los espaciales y ornamentales interiores de las obras presentadas.

Durante la primera mitad del siglo XVI, la isla Española fue eje de la conquista, colonización y evangelización de Nuevo Mundo en especial la ciudad de Santo Domingo donde se instalaron las estructuras gubernamentales, monárquicas y religiosas más importantes, centralizadas bajo el control absoluto de la corona de España. Así pues, para llevar el control se necesitaba de instituciones que estuvieran en manos de personas de entera confianza de los Reyes Católicos. (P.29)

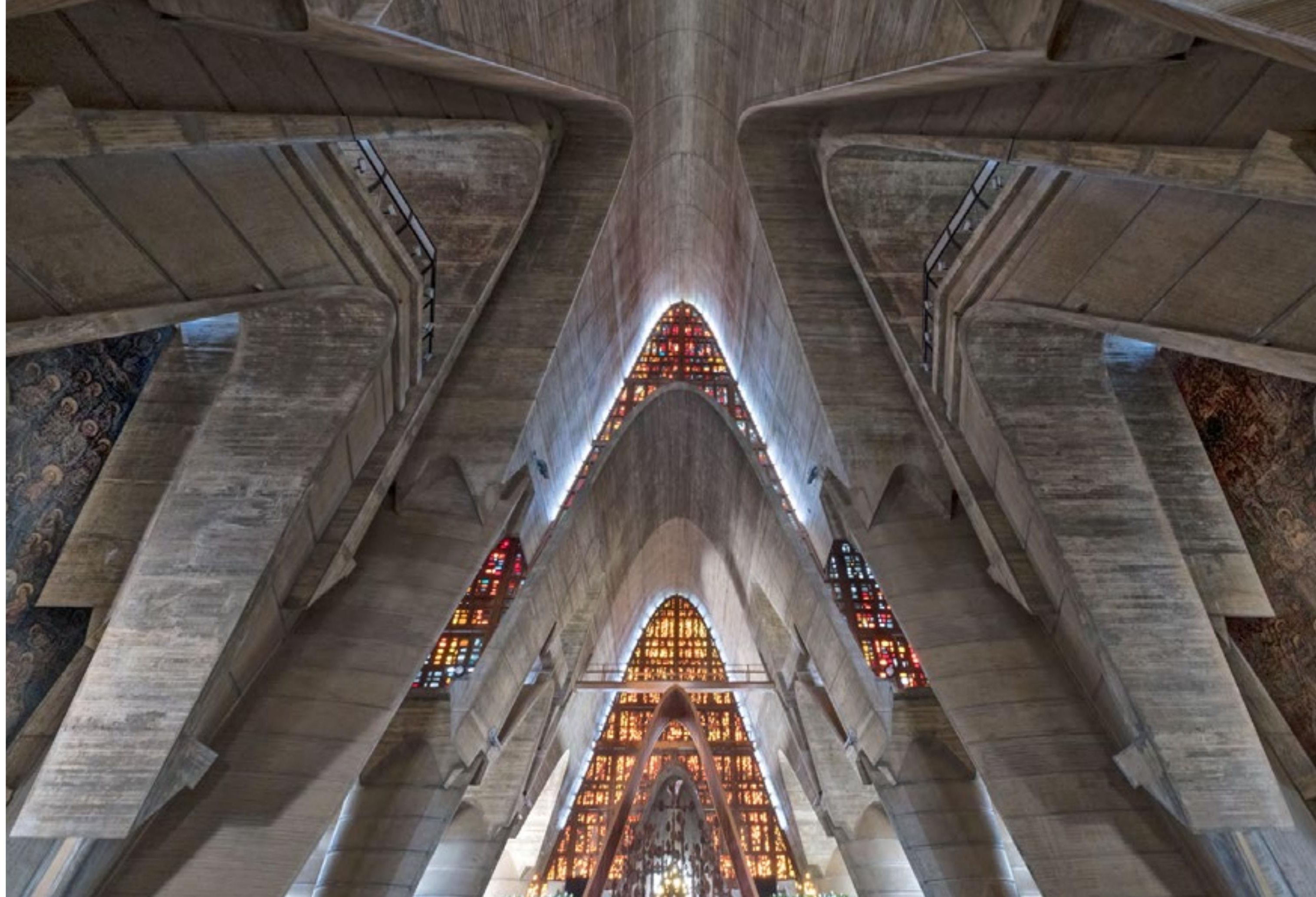


Un matiz crítico podría ser que a pesar de que la introducción propone un acercamiento a los espacios de devoción como puntos de unión de la nación dominicana, no se profundiza en su rol contemporáneo y su vínculo con los fieles y la nación en general en la actual situación de pandemia. El objetivo de lograr un producto que permita una mejor difusión y promoción de los edificios religiosos en República Dominicana con un objetivo de valoración cultural, patrimonial y turístico es alcanzado ampliamente por la publicación. Esta observación no resta valor a una que permite seguir difundiendo las investigaciones sobre arquitectura colonial en América Latina.

El libro "Un pueblo unido por la fé: espacios de devoción" está disponible a través de la aplicación MIRA (Mi Realidad Aumentada) que está disponible para descargar en www. popularenlinea.com/mira. El libro se complementa con un documental homónimo. 
Doctora en Arquitectura por la Universidad Michoacana de San Nicolás de Hidalgo (UMSNH). Morelia, México (2006). Maestría en Conservación y Restauración de Monumentos y Bienes Culturales, Universidad Nacional Pedro Henríquez Ureña (UNPHU), Santo Domingo. R. D. (2001) Arquitecta (Cum Laude), Universidad Iberoamericana (UNIBE), Santo Domingo, República Dominicana (1989). Especialidad en Cast Stone and Historic Concrete Rehabilitation. The (APTI), Denver, Colorado, Estados Unidos (2010). Especialidad en Cooperación Internacional al Desarrollo, Especialdad en Cooperación Internacional al Desarrollo, Clearing Masonry Structure, RESTORE, Seattle, Estados Unidos (2002).
Doctor en Arquitectura con mención Honorífica. Universidad Michoacana, México (2008). Maestría en Conservación de Monumentos y Bienes Culturales. Universidad Nacional Pedro Henríquez Ureña, Santo Domingo, República Dominicana (2003). Arquitecto mención de honor "Cum Laude", Universidad Naciona Pedro Henríquez Ureña, Santo Domingo, República Dominicana (1972).

Fue Director Fundador del Centro de Inventario de Bienes Culturales: Director Nacional de Patrimonio Cultural de 1986 a 1996; Miembro Fundador y de Honor del Comite 1986 a 1996; Miembro Fundador y de Honor del Comite Dominicano del ICOMOS; Vicepresidente Mundial de
ICOMOS de 1993 a 1999; Miembro Fundador del ICOM-DO y de CARIMOS, Miembro de la Association for Preservation Tecnology International, y de la Sociedad Española de Tecnology International, y de la Sociedad Española de Director de la Oficina de la Obra y Museos de la Catedral de Santo Domingo y Director del Centro de Altos Estudios Humanísticos y del Idioma Español, adscrito a la UNPHU. 